\title{
ISOLAMENTO E AVALIAÇÃo DA ATIVIDADE NEMATICIDA DE CONSTITUINTES QUÍMICOS DE Mucuna cinerea CONTRA Meloidogyne incognita E Heterodera glycines
}

Antonio Jacinto Demuner*, Luiz Cláudio de Almeida Barbosa, Jeferson Chagas do Nascimento e Jessy James Vieira

Departamento de Química, Universidade Federal de Viçosa, 36571-000 Viçosa - MG

Maria Amelia dos Santos

Departamento de Agronomia, Universidade Federal de Uberlândia, 38400-902 Uberlândia - MG

Recebido em 27/5/02; aceito em 8/10/02

ISOLATION AND NEMATOCIDAL ACTIVITY EVALUATION OF CHEMICAL CONSTITUENTS FROM Mucuna cinerea AGAINST Meloidogyne incognita AND Heterodera glycines. Phytochemical investigation of the aerial parts and roots of Mucuna cinerea led to the isolation of a mixture of fatty acids, triacylglicerols, $\beta$-sitosterol, stigmasterol, stigmasterol glucoside, daucosterol, asperglaucide (4) and the isoflavonoids prunetin (1), genistein (2), medicarpin (3), daidzein (5), 7-O- $\alpha$-glycopiranosyl daidzein (6). An in vitro bioassay was carried out with compounds $\mathbf{1 - 4}$, at the concentration of 50 and $5 \mu \mathrm{g} \mathrm{mL}^{-1}$ against the phytonematodes $M$. incognita and $H$. glycines. Although the four compounds showed some nematocidal property, the most active was (1), causing $70 \%$ mortality of $M$. incognita at the concentration of $50 \mu \mathrm{gL}^{-1}$.

Keywords: nematodes; natural nematicides; velvet bean.

\section{INTRODUÇÃO}

O gênero Mucuna (Leguminosae) compreende aproximadamente 160 espécies. No Brasil, as espécies mais comumente encontradas são M. deeringiana, M. utilis, M. pruriens, M. cochichinensis, $M$. nivea, M. capitata, $M$. hassjoo, $M$. diabolica, $M$. aterrima e $M$. cinerea sendo que as principais diferenças entre as espécies cultivadas são as características da pubescência da vagem, a cor da semente e o tempo para a colheita da vagem ${ }^{1}$. Estas espécies são muito utilizadas para adubação verde e para silagem, devido à grande quantidade de matéria orgânica de alta digestibilidade que produz e de sua capacidade de controlar fitonematóides ${ }^{1-4}$.

Poucos estudos químicos foram realizados neste gênero, sendo identificados isoflavonóides, antocianinas e antocianidinas ${ }^{5}, \mathrm{C}$ glicosilflavonas ${ }^{6}$ e L-Dopa ${ }^{7}$. De $M$. aterrima foram isolados ésteres e ácidos graxos, esteróides e alantoína ${ }^{8-10}$. Suas sementes são constituídas principalmente de amido (40\%), proteínas (17\%) e lipídios $(1,8 \%)^{11}$.

O uso de Mucuna como controladora de fitonematóides ${ }^{12-14}$ esti- $^{-}$ mulou o estudo químico de Mucuna cinerea e a avaliação da atividade nematicida sobre Meloidogyne incognita e Heterodera glycines, das substâncias descritas pela primeira vez no gênero Mucuna.

\section{PARTE EXPERIMENTAL}

\section{Procedimentos experimentais gerais}

As temperaturas de fusão foram determinadas em aparelho Microquímica MQAPF-301. Os espectros na região do infravermelho foram obtidos em um espectrômetro Perkin Elmer FTIR 1000, na região de 600 a $4000 \mathrm{~cm}^{-1}$. Os espectros de ressonância magnética nuclear de hidrogênio $\left(\mathrm{RMN}{ }^{1} \mathrm{H}\right)$ e de carbono $13\left(\mathrm{RMN}{ }^{13} \mathrm{C}\right)$ a 400 e $100 \mathrm{MHz}$, respectivamente, foram obtidos em aparelho Bruker WM 400 ou DRX 400. Para as separações cromatográficas em coluna, utilizou-se sílica gel 60 (70 - 230 Mesh-ASTM, Merck). Para as

*e-mail: ademuner@ufv.br
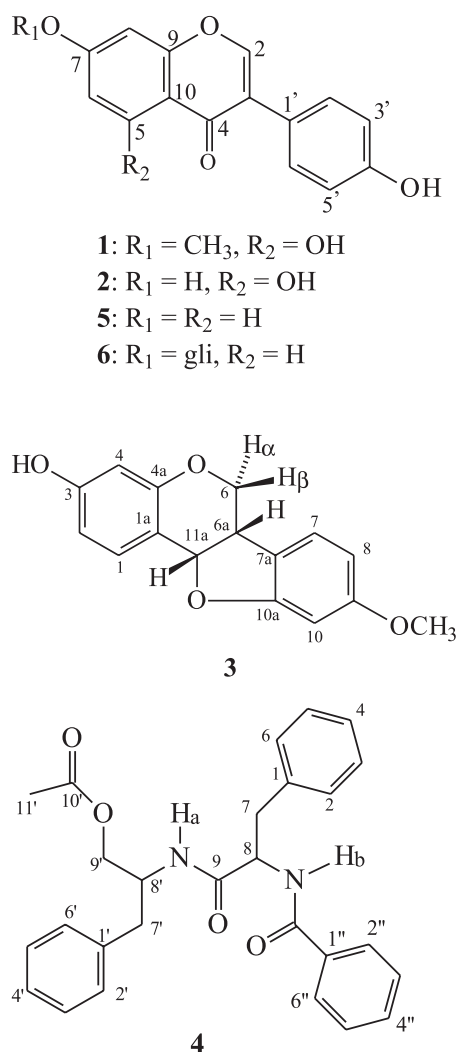

4

Figura 1. Compostos isolados de M. cinerea

análises cromatográficas em camada delgada, utilizaram-se placas da Merck Kieselgel $60 \mathrm{~F}_{254}(0,25 \mathrm{~mm}$ de sílica gel com indicador de fluorescência $\left.\mathrm{UV}_{254}\right)$. Os cromatogramas dos ésteres metílicos foram obtidos em um cromatógrafo Shimadzu GC 17-A equipado com um injetor automático (AOC 17), um detector de ionização de chama (FID) e uma coluna capilar Carbowax 20M (30 m x 0,25 mm), 
filme de $0,25 \mu \mathrm{m}$. O fluxo do gás de arraste $\left(\mathrm{N}_{2}\right)$ foi de $1,0 \mathrm{~mL} / \mathrm{min}$. As temperaturas utilizadas foram $200{ }^{\circ} \mathrm{C}$ para o injetor, $250{ }^{\circ} \mathrm{C}$ para o detector e, inicialmente, $150{ }^{\circ} \mathrm{C}$ para a coluna, com programação de aquecimento de $3{ }^{\circ} \mathrm{C} / \mathrm{min}$ até atingir $200{ }^{\circ} \mathrm{C}$, permanecendo nesta temperatura por $40 \mathrm{~min}$.

\section{Preparo e quantificação dos ésteres metílicos por cromatografia em fase gasosa}

A preparação dos ésteres metílicos a partir dos ésteres e ácidos graxos, para análise por cromatografia em fase gasosa, foi realizada de acordo metodologia descrita por Paula et al. ${ }^{15}$.

\section{Material vegetal}

A espécie Mucuna cinerea foi coletada em Viçosa, MG, em 1997. A exsicata encontra-se depositada no Herbário do Departamento de Biologia Vegetal da Universidade Federal de Viçosa, sob o número 9248 .

\section{Extração e purificação}

Amostras, secas ao ar, de raiz $(710 \mathrm{~g})$ e caule $(760 \mathrm{~g})$ de $M$. cinerea foram moídas e submetidas à extração com etanol, a quente, em aparelho Soxhlet, durante $72 \mathrm{~h}$. A remoção do solvente, por destilação em evaporador rotatório, sob pressão reduzida, levou à obtenção de $41 \mathrm{~g}$ de extrato da raiz e $140 \mathrm{~g}$ do caule.

$\mathrm{O}$ extrato do caule foi submetido a sucessivos fracionamentos cromatográficos em coluna de sílica gel utilizando-se misturas de hexano-éter dietílico de polaridade crescente, sendo posteriormente agrupadas em 8 frações. A fração 1 (5,6 g) apresentou-se como um óleo amarelo pálido, caracterizado por infravermelho como sendo constituída de uma mistura de triacilgliceróis. Esta foi então submetida à reação de transesterificação com $\mathrm{MeOH} / \mathrm{MeONa}{ }^{16}$ fornecendo os ésteres metílicos correspondentes. A fração 2 (3,4 g), constituída de uma mistura de ácidos graxos, foi submetida à reação de esterificação com $\mathrm{BF}_{3} / \mathrm{MeOH}^{15} \mathrm{e}$ os ésteres metílicos obtidos, assim como os da fração 1 , foram analisados por cromatografia em fase gasosa.

As demais frações apresentaram-se como sólidos brancos, sendo a fração $3(0,49 \mathrm{~g})$ caracterizada como uma mistura de $\beta$-sitosterol + estigmasterol e a fração 4 (0,020 g), como uma mistura de daucosterol + estigmasterol glicosilado. As demais frações (5 a 8) apresentaram-se como compostos puros, sendo identificadas como: fração $5(0,017 \mathrm{~g})$, prunetina (1); fração $6(0,041 \mathrm{~g})$, genisteína (2); fração 7 (0,037 g), medicarpina (3) e fração $8(0,044 \mathrm{~g})$, asperglaucídeo (4).

O extrato etanólico da raiz foi também submetido a sucessivas separações cromatográficas em coluna de sílica gel utilizando-se misturas de hexano-acetato de etila de polaridade crescente. Esse procedimento resultou em três frações ( 9 a 11) que tiveram seus constituintes químicos caracterizados. A fração 9 foi caracterizada como uma mistura de ésteres graxos e, portanto, submetida ao mesmo procedimento da fração 1 . A fração $10(0,080 \mathrm{~g})$ foi recristalizada em clorofórmio-metanol fornecendo a daidzeína (5) como um sólido amarelado. A fração $11(0,100 \mathrm{~g})$ também foi recristalizada em clorofórmio-metanol, resultando na obtenção de um sólido amarelo, identificado como daidzeína-7-O- $\alpha$-glicopiranosídeo (6).

$\beta$-sitosterol, estigmasterol, daucosterol e estigmasterol glicosilado

Os dados espectroscópicos (IV, RMN de ${ }^{1} \mathrm{H}$ e de ${ }^{13} \mathrm{C}$ e EM) e as temperaturas de fusão desses compostos foram similares àqueles registrados na literatura ${ }^{15,17}$.
Prunetina (4',5-diidroxi-7-metoxiisoflavona) (1): Tf. 235$237{ }^{\circ} \mathrm{C}$ (lit. $\left.{ }^{18} 240{ }^{\circ} \mathrm{C}\right)$; IV $v_{\text {máx }}(\mathrm{KBr}) \mathrm{cm}^{-1} 3384,3050,2960,1668$, $1614,1570,1518,1358,1030,800$; RMN ${ }^{1} \mathrm{H}(400 \mathrm{MHz}$, DMSO$\left.\mathrm{d}_{6}\right) \delta 3,86\left(\mathrm{~s}, 7-\mathrm{OCH}_{3}\right), 6,41(\mathrm{~d}, J=2,2 \mathrm{~Hz}, \mathrm{H}-6), 6,65(\mathrm{~d}, J=2,2 \mathrm{~Hz}$, H-8), 6,82 (d, $J=8,6$ Hz, H-5' e H-3'), 7,39 (d, $J=8,6$ Hz, H-6' e H$\left.2^{\prime}\right), 8,40$ (s, H-2), 9,60 (s, HO-4'), 12,96 (s, HO-5); RMN ${ }^{13} \mathrm{C}(100$ $\left.\mathrm{MHz}, \mathrm{DMSO}-\mathrm{d}_{6}\right) \delta$ 55,9 (H $\left.\mathrm{CO}-7\right), 92,3$ (C-8), 97,9 (C-6), 105,4 (C-10), 115,0 (C-3' e C-5'), 121,0 (C-1'), 122,4 (C-3), 130,0 (C-2' e C-6'), 154,3 (C-2), 157,4 (C-9), 157,5 (C-4'), 161,7 (C-5), 165,2 (C-7), 180,3 (C-4).

Genisteína (4',5,7-triidroxiisoflavona) (2): Tf. 296-297 ${ }^{\circ} \mathrm{C}$ (lit..$^{18}$ 297-298 $\left.{ }^{\circ} \mathrm{C}\right)$; IV $v_{\text {máx }}(\mathrm{KBr}) \mathrm{cm}^{-1} 3440,3050,1655,1577,1516,1370$, 1244, 1046; RMN ${ }^{1} \mathrm{H}\left(400 \mathrm{MHz}, \mathrm{DMSO}-\mathrm{d}_{6}\right) \delta 6,24(\mathrm{~d}, J=2,2 \mathrm{~Hz}$, H-6), 6,39 (d, $J=2,2 \mathrm{~Hz}, \mathrm{H}-8), 6,82$ (d, $J=8,6 \mathrm{~Hz}, \mathrm{H}-3$ ' е H-5'), 7,39 (d, $J=8,6$ Hz, H-2' e H-6'), 8,40 (s, H-2), 9,60 (s, HO-4'), 10,8 (s, HO-7), 12,96 (s, HO-5); RMN ${ }^{13} \mathrm{C}\left(100 \mathrm{MHz}, \mathrm{DMSO}-\mathrm{d}_{6}\right) \delta 94,0$ (C-8), 99,0 (C-6), 104,4 (C-10), 115,0 (C-3' e C-5'), 121,2 (C-1'), 122,3 (C-3), 130,1 (C-2' e C-6'), 154,2 (C-2), 157,4 (C-4'), 157,6 (C-9), 162,0 (C-5), 165,3 (C-7), 180,2 (C-4).

Medicarpina (3-hidroxi-9-metoxipterocarpano) (3): Tf. 127$129{ }^{\circ} \mathrm{C}$ (lit. $\left.{ }^{19} 127,5-128,5^{\circ} \mathrm{C}\right)$; IV v $v_{\text {máx }}(\mathrm{KBr}) \mathrm{cm}^{-1} 3400,3070,2950$, 2880, 1640, 1600, 1500, 1475, 1350, 1270, 1030, 950, 800; RMN ${ }^{1} \mathrm{H}\left(400 \mathrm{MHz}, \mathrm{CDCl}_{3}\right) \delta 3,52$ (ddd, $J=6,7,10,9$ e 4,8 Hz, H-6a),

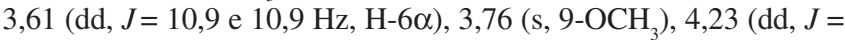
10,9 e 4,8 Hz, H-6b), 5,06 (s, HO-3), 5,48 (d, $J=6,7$ Hz, H-11a), 6,40 (d, $J=2,5$ Hz, H-10), 6,43-6,45 (m, H-4 e H-8), 6,54 (dd, $J=$ 8,3 e 2,2 Hz, H-2), 7,12 (d, $J=9$ Hz, H-7), 7,37 (d, $J=8,3$ Hz, H-1); $\mathrm{RMN}{ }^{13} \mathrm{C}\left(100 \mathrm{MHz}, \mathrm{CDCl}_{3}\right) \delta 39,7(\mathrm{C}-6 \mathrm{a}), 55,4\left(\mathrm{OCH}_{3}\right), 66,4(\mathrm{C} 6)$, 78,4 (C-11a), 96,8 (C-10), 103,6 (C-4), 106,3 (C-8), 109,6 (C-2), 112,5 (C-1a), 119,0 (C-7a), 124,6 (C-7), 132,1 (C-1), 156,5 (C-4a), 156,9 (C-3), 160,5 (C-10a), 161,0 (C-9).

Asperglaucídeo (acetato de $N$-benzoilfenilalanil-L-fenilalaninol) (4): Tf. $185-186^{\circ} \mathrm{C}$ (lit. ${ }^{20} 187-188^{\circ} \mathrm{C}$ ); IV v ${ }_{\text {máx }}(\mathrm{KBr}) \mathrm{cm}^{-1} 3312,3032$, 2924, 2860, 1726, 1661, 1632; RMN ${ }^{1} \mathrm{H}\left(400 \mathrm{MHz}_{\max } \mathrm{CDCl}_{3}\right) \delta 2,01$ (s, H-11'), 2,72 (dd, $J=14$ e 7,4 Hz, Ha-7'), 2,75 (dd $J=14$ e 7,4 $\mathrm{Hz}, \mathrm{Hb}-7$ '), 3,04 (dd, $J=13,4$ e 8,4 Hz, Ha-7), 3,21 (dd, $J=13,4$ e 6,1 Hz, Hb-7), 3,80 (dd, $J=11,6$ e 4,2 Hz, Ha-9'), 3,96 (dd, $J=11,6$ e 4,8 Hz, Hb-9'), 4,29-4,36 (m, H-8'), 4,75 (ddd, $J=8,4,7,4$ e 6,1 Hz, H-8), 5,96 (d, $J=7,4$ Hz, H-b), 6,70 (d, $J=8,7$ Hz, H-a), 7,08 (d, $J=5,4$ Hz, H-2' e H-6'), 7,11-7,17 (m, H-3', H-4', H-5'), 7,227,29 (m, 5H, H-2a H-6), 7,42 (m, H-3" e H-5”), 7,51 (tt, H-4”), 7,69 (dt, H-2" e H-6").

Daidzeína (4',7-diidroxiisoflavona) (5): Tf. $312^{\circ} \mathrm{C}$ (decompõe) (lit. ${ }^{18} 315{ }^{\circ} \mathrm{C}$ ); IV $v_{\text {máx }}(\mathrm{KBr}) \mathrm{cm}^{-1} 3500,3220,1610,1570,1520$, $1490,1280,1240,1115,800 ; \mathrm{RMN}^{1} \mathrm{H}\left(400 \mathrm{MHz}, \mathrm{DMSO}-\mathrm{d}_{6}\right) \delta$ 6,82 (d, $J=8,8 \mathrm{~Hz}, \mathrm{H}-3$ ' e H-5'), 6,87 (d, $J=2,1 \mathrm{~Hz}, \mathrm{H}-8$ ), 6,94 (dd, $J=8,8$ e $2,1 \mathrm{~Hz}, \mathrm{H}-6), 7,39$ (d, $J=8,8 \mathrm{~Hz}, \mathrm{H}-2$ ' e H-6'), 7,98 (d, $J$ $=8,8 \mathrm{~Hz}, \mathrm{H}-5), 8,28$ (s, H-2), 9,54 (s, HO-4'), 10,78 (s, HO-7); RMN ${ }^{13} \mathrm{C}\left(100 \mathrm{MHz}, \mathrm{DMSO}-\mathrm{d}_{6}\right) \delta 102,1$ (C-8), 115,0 (C-3' e C-5'), 115,2 (C-6), 116,7 (C-10), 122,6 (C-3), 123,6 (C-1'), 127,8 (C-5), 130,1 (C-2' e C-6'), 152,3 (C-2), 157,2 (C-4'), 157,5 (C-7), 162,6 (C-9), $174,8(\mathrm{C}-4)$.

Daidzeína-7-O- $\alpha$-glicopiranosídeo (6): Tf. 229-231 ${ }^{\circ} \mathrm{C}$ (lit. ${ }^{18}$ $\left.234{ }^{\circ} \mathrm{C}\right)$; IV $v_{\text {máx }}(\mathrm{KBr}) \mathrm{cm}^{-1} 3550-3300,1610,1570,1520,1490$, $1280,1240,1110,1050,800 ; \mathrm{RMN}^{1} \mathrm{H}\left(400 \mathrm{MHz}, \mathrm{CD}_{3} \mathrm{OD}\right) \delta 3,44-$ 3,49 (m, H-4"), 3,55-3,57 (m, H-2” ), 3,58-3,62 (m, H-5"), 3,703,71 (m, H-3"), 3,76 (dd, $J=12,1$ e 2,4 Hz, Ha-6"), 3,96 (dd, $J=$ 12,1 e 5,7 Hz, Hb-6”), 5,14 (d, $J=7,7$ Hz, H-1"), 6,89 (d, $J=8,8 \mathrm{~Hz}$, 
H-3' e H-5'), 7,25 (dd, $J=8,8$ e 2,1 Hz, H-6), 7,28 (d, $J$ := 2,1, H-8), 7,42 (d, $J=8,8$ Hz, H-2' e H-6'), 8,18 (d, $J=8,8$ Hz, H-5), 8,21 (s, $\mathrm{H}-2), 9,54$ (s, HO-4'); RMN ${ }^{13} \mathrm{C}$ (100 MHz, CD OD) $\delta$ 62,6 (C-6"), 71,4 (C-4”), 74,8 (C-2”), 77,9 (C-3”), 78,4 (C-5”), 101,9 (C-1”), 102,1 (C-8), 115,0 (C-3' e C-5'), 115,2 (C-6), 116,7 (C-10), 122,6 (C-3), 123,5 (C-1'), 127,8 (C-5), 131,4 (C-2' e C-6'), 154,9 (C-2), 158,9 (C-4'), 159,2 (C-7), 163,5 (C-9), 178,6 (C-4).

\section{Ensaio biológico}

Para a avaliação da atividade nematicida dos compostos 1-4, in vitro, os ensaios foram realizados com as espécies $M$. incognita e $H$. glycines, utilizando-se a metodologia do tubo invertido, ${ }^{9}$. Os ensaios foram realizados nas concentrações de 5 e $50 \mu \mathrm{g} \mathrm{mL}^{-1}$, ao acaso e com 6 repetições.

\section{RESULTADOS E DISCUSSÃO}

A fração 1, obtida do caule, foi caracterizada pelo seu espectro no infravermelho como uma mistura de ésteres graxos. $\mathrm{O}$ tratamento dessa fração com $\mathrm{MeONa} / \mathrm{MeOH}$ e posterior análise por cromatografia em fase gasosa (CG) resultou na identificação dos ésteres metílicos dos seguintes ácidos: mirístico (C14:0, 4\%), palmítico (C16:0, 61\%), esteárico (C18:0, 13\%), oléico (C18:1, 7\%), linoléico (C18:2, 4\%) e icosanóico (C20:0, 2\%).

A fração 9, obtida da raiz, apresentou-se semelhante à fração 1, sendo identificados os mesmos ésteres metílicos, aproximadamente nas mesmas proporções.

O espectro no infravermelho da fração $2(3,4 \mathrm{~g})$ apresentou bandas de absorção típicas de ácidos graxos (3600-2500 cm-1 ( $\mathrm{V} \mathrm{OH}$ ); $1710 \mathrm{~cm}^{-1}(\vee \mathrm{CO}), 720 \mathrm{~cm}^{-1}\left(\delta\left[\mathrm{CH}_{2}\right]_{\mathrm{n}}, \mathrm{n}>4\right)$. A esterificação dessa fração com $\mathrm{BF}_{3} / \mathrm{MeOH}$, seguida de análise por $\mathrm{CG}$, permitiu a identificação dos ésteres metílicos dos ácidos graxos: mirístico (C14:0, 2\%), palmítico (C16:0, 42\%), esteárico (C18:0, 10\%), oléico (C18:1, 9\%), linoléico (C18:2, $24 \%)$, linolênico $(\mathrm{C} 18: 3,11 \%)$ e icosanóico (C20:0, 1\%).

A fração $3(0,5 \mathrm{~g})$ foi identificada como uma mistura de $\beta$ sitosterol e estigmasterol, na proporção de 1:1, conforme análise do seu espectro de $\mathrm{RMN}{ }^{1} \mathrm{H}$.

A fração $4(0,02 \mathrm{~g})$ foi identificada como uma mistura de daucosterol + estigmasterol $\beta$-D-glicosídeo, por comparação com uma amostra padrão e, ainda, por comparação de seus dados espectroscópicos com os da literatura ${ }^{15}$. A análise de seu espectro de RMN ${ }^{1} \mathrm{H}$ revelou uma proporção aproximada de 9:1, de seus respectivos constituintes.

A fração 5 apresentou-se como um sólido amarelo correspondente ao composto 1 . No seu espectro de $\mathrm{RMN}{ }^{13} \mathrm{C}$ pode-se verificar a presença de sinais referentes à carbonila $(\delta 180,3)$ e metoxila $(\delta$ 55,9). No espectro de $\mathrm{RMN}{ }^{1} \mathrm{H}$, observou-se um singleto em $\delta 12,96$ atribuído à hidroxila HO-5 e outro em $\delta 9,60$, atribuído à hidroxila HO-4'. Observou-se também a presença de dois dubletos em $\delta 7,39$ e $\delta 6,82$, ambos integrados para dois hidrogênios, sendo estes atribuídos aos hidrogênios H-2' e H-6' $(J=8,6 \mathrm{~Hz})$ e aos hidrogênios H-3' e H-5' ( $J=8,6 \mathrm{~Hz})$, respectivamente. Já o singleto em 3,86 $\delta$, integrado para três hidrogênios, foi atribuído aos hidrogênios da metoxila.

A proximidade espacial da hidroxila (HO-4') com os hidrogênios H-5' e H-3', bem como dos hidrogênios H-6 e H-8 com a metoxila do carbono 7 , foi determinada por experimentos de nOe (Tabela 1). Através destes, pode-se também verificar a proximidade entre H-2' e H-2.

O composto 1, conhecido como prunetina, é encontrado em diversas espécies de leguminosas do gênero Prunus, como $P$.
Tabela 1. Efeito nuclear Overhause (nOe) observado para alguns hidrogênios irradiados nos isoflavonóides $\mathbf{1}$ e $\mathbf{2}$

\begin{tabular}{lll}
\hline H irradiados & \multicolumn{2}{c}{ nOe observados } \\
\cline { 2 - 3 } & $\mathbf{1}$ & $\mathbf{2}$ \\
\hline $\mathrm{HO}-5$ & $\mathrm{H}-6$ & $\mathrm{H}-6$ \\
$\mathrm{H}-2$ & $\mathrm{H}-{ }^{\prime}$, & $\mathrm{H}-2^{\prime}$, \\
$\mathrm{HO}-4$, & $\mathrm{H}-5,, \mathrm{H}-3$ & $\mathrm{H}-5^{\prime}, \mathrm{H}-3$ \\
$\mathrm{CH}_{3} \mathrm{O}-7$ & $\mathrm{H}-6, \mathrm{H}-8$ & - \\
$\mathrm{HO}-7$ & - & $\mathrm{H}-6, \mathrm{H}-8$ \\
$\mathrm{H}-8$ & $\mathrm{CH}_{3} \mathrm{O}-7$ & $\mathrm{HO}-7$ \\
$\mathrm{H}-6$ & $\mathrm{CH}_{3} \mathrm{O}-7, \mathrm{HO}-5$ & $\mathrm{HO}-7, \mathrm{HO}-5$ \\
\hline
\end{tabular}

aequinoctialis, P. nipponica, P. maximowiczii e $P$. avium ${ }^{22}$. A prunetina também foi isolada da bactéria Mycobacterium phlei ${ }^{23}$. No entanto, não foi verificado, na literatura, qualquer relato sobre a sua ocorrência no gênero Mucuna.

Da fração $6(0,041 \mathrm{~g})$, foi isolada a genisteína (2) que, apesar de ser uma substância amplamente distribuída em plantas ${ }^{22,24}$ e outros organismos vivos ${ }^{23}$, a sua ocorrência no gênero Mucuna está sendo originalmente descrita neste trabalho.

$\mathrm{O}$ espectro de RMN ${ }^{1} \mathrm{H}$ do composto 2 apresentou-se bastante semelhante ao do composto 1 . A principal diferença é a ausência do sinal referente à metoxila e a presença de um outro sinal alargado em $\delta 10,8$, atribuído à hidroxila (HO-7). As atribuições dos sinais no espectro de $\mathrm{RMN}{ }^{1} \mathrm{H}$ foram confirmadas pelos experimentos de nOe (Tabela 1).

O espectro de RMN ${ }^{13} \mathrm{C}$ da fração 7 (0,017 g, composto 3$)$ mostrou um total de 16 sinais, sendo observados sinais em $\delta 66,4$, atribuído ao carbono metilênico C-6, e em $\delta 55,4$, atribuído à metoxila. $\mathrm{O}$ espectro de $\mathrm{RMN}{ }^{1} \mathrm{H}$ apresentou sinais correspondentes a $14 \mathrm{hi}-$ drogênios. $\mathrm{O}$ singleto em $\delta 3,76$, integrado para três hidrogênios, foi atribuído à metoxila $\left(9-\mathrm{OCH}_{3}\right)$ e o sinal largo em $\delta 5,06$ foi atribuído à hidroxila $(3-\mathrm{OH})$.

Através dos experimentos de nOedif foi possível confirmar a localização da metoxila e da hidroxila nos anéis benzênicos e, também, a estereoquímica cis dos hidrogênios H-6a e H-11a. Quando H-11a foi irradiado, observou-se nOe em H-6a $(5,7 \%)$ e H-1 $(3,4 \%)$. Quando H-6a foi irradiado, observou-se nOe em H-11a (5,7\%), H$6 \beta(5,7 \%)$ e H-7 (1,4\%). Ao irradiar H-6 $\alpha$ observou-se um maior

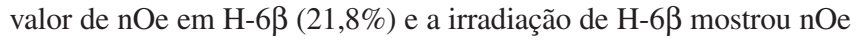
de $5,7 \%$ em H-6a e $21,8 \%$ em H-6 $\alpha$.

O composto 3, conhecido como medicarpina, já foi isolado de algumas leguminosas, como Andira inermis, Dalbergia stevensonii, D. nitidula, D. variabilis e Swartzia madagascariensis ${ }^{19,25,26}$, porém, não foi encontrado registro do isolamento do mesmo em plantas do gênero Mucuna.

O espectro no infravermelho da fração 8 (composto 4) apresentou uma forte banda de absorção em $3312 \mathrm{~cm}^{-1}(\mathrm{O}-\mathrm{H})$, além de absorções em $3032 \mathrm{~cm}^{-1}$ (C-H de carbono insaturado), em $1726 \mathrm{~cm}^{-1}$ ( $\mathrm{C}=\mathrm{O}$ de éster) e em $1632 \mathrm{~cm}^{-1}$ ( $\mathrm{C}=\mathrm{O}$ de amida).

No espectro de $\mathrm{RMN}{ }^{13} \mathrm{C}$, verificou-se a presença de três sinais, em $\delta 170,7,170,1$ e 167,0, referentes às carbonilas, além de 15 sinais de carbonos aromáticos compreendidos entre $\delta 126$ e 130 .

Pela análise do seu espectro de $\mathrm{RMN}{ }^{1} \mathrm{H}$, pode-se observar a presença de um singleto em $\delta 2,01$, integrado para três hidrogênios, que foi atribuído ao grupo $\mathrm{CH}_{3}-\mathrm{CO}$. Os dubletos alargados em $\delta 5,96$ e 6,70 , integrados para um hidrogênio cada, foram atribuídos aos hidrogênios $\mathrm{H}-\mathrm{a}$ e H-b, respectivamente. Pela análise desse espectro pode-se observar a presença de sinais de hidrogênios aromáticos na região de $\delta 7,10-7,80$. A análise de todos os dados espectros- 
cópicos resultou na proposição da estrutura $\mathbf{4}$ para o composto dessa fração.

Através do experimento de nOedif (Tabela 2), pode-se observar a proximidade espacial dos hidrogênios dos anéis aromáticos com os hidrogênios H-b, H-7, H-8, H-8' e H-9', bem como a proximidade dos hidrogênios H-b com H-8 e H-a com H-8' e H-8.

Tabela 2. Dados de nOe observados para alguns hidrogênios irradiados no composto 4

\begin{tabular}{|c|c|}
\hline $\mathrm{H}$ irradiados & nOe observados $(\%)$ \\
\hline H-8, & H-9' $(1,8)$, H-7' $(4,9), \mathrm{H}-\mathrm{a}(2,4), \mathrm{H}-2$ ' е H-6' $(1,3)$ \\
\hline $\mathrm{H}-8$ & H-7 $(3,5)$, H-a $(6,9)$, H-b $(2,3)$ \\
\hline $\mathrm{H}-\mathrm{a}$ & H-8 $(6,9), H-8,(2,4)$ \\
\hline $\mathrm{H}-\mathrm{b}$ & H-8' $(3,7), H-8(2,3)$ \\
\hline $\mathrm{H}-7$ & H-8' (4,9), H-2' e H-6' $(2,6)$, H-9' (1) \\
\hline H-2" e H-6" & $\mathrm{H}-\mathrm{b}(3,7)$ \\
\hline $\mathrm{H}-2$ e $\mathrm{H}-6$ & H-7 $(3,2), H-8(1)$ \\
\hline H-2' e H-6' & H-8' $(1,3), \mathrm{H}^{\prime} 7^{\prime}(2,6)$ \\
\hline
\end{tabular}

O composto 4, conhecido como asperglaucídeo, foi primeiramente isolado do fungo Aspergillus glaucus ${ }^{26}$ e, também, da alga Cystoseira corniculata Hauck $^{20}$. Entretanto, o mesmo ainda não havia sido identificado em leguminosas do gênero Mucuna.

As frações 10 e 11 foram obtidas a partir das raízes, sendo ambas constituídas de sólidos amarelos claro. Pela análise dos dados de RMN ${ }^{1} \mathrm{H}$ e ${ }^{13} \mathrm{C}$, e comparação com os dados da literatura ${ }^{18}$, foi possível identificar, inequivocamente, os constituintes dessas frações como os isoflavonóides daidzeína (5) e daidzeína 7-O-Q-glicopiranosídeo (6).
Avaliação da atividade nematicida dos compostos isolados de M. cinerea

No primeiro ensaio, com as amostras (compostos 1-4) na concentração de $50 \mu \mathrm{g} \mathrm{mL}^{-1}$ observou-se que apenas dois compostos causaram taxa de mortalidade superior a $50 \%$ para $M$. incognita, sendo estes a prunetina $(\mathbf{1}, 70 \%)$ e a genisteína $(\mathbf{2}, 57 \%)$. A análise de variância confirmou o efeito significativo das amostras sobre os juvenis de $M$. incognita. Para a mesma concentração $\left(50 \mu \mathrm{g} \mathrm{mL}^{-1}\right)$, no ensaio com o nematóide $H$. glycines, nenhum dos compostos testados apresentou efeito significativo (Tabela 3 ).

No segundo ensaio, utilizando as amostras com concentração final de $5 \mu \mathrm{g} \mathrm{mL} \mathrm{g}^{-1}$, observou-se redução nas taxas de mortalidade. Com o nematóide $M$. incognita apenas o asperglaucídeo (4) não causou mortalidade significativa em relação ao controle. Para $H$. glycines, nenhum dos compostos testados na concentração de $5 \mu \mathrm{g} \mathrm{mL}^{-1}$ causou efeito significativo sobre a mortalidade dos juvenis (Tabela 3).

Estes resultados mostram que, em relação ao nematóide $M$. incognita, mais de uma classe de compostos (isoflavonóide e amida) está associada à atividade nematicida.

Considerando que os compostos ativos isolados, no presente trabalho, encontram-se em quantidade muito pequena na planta, e que a mesma apresenta comprovada ação nematicida no campo ${ }^{1-4,27}$, é possível que esse efeito seja devido não somente a estas substâncias, mas também, a outras substâncias presentes na mesma, que não foram identificadas, ou ainda, a substâncias comuns, tais como $\beta$ sitosterol, estigmasterol, ácidos e ésteres graxos, presentes em maior concentração e que também apresentam acentuada atividade nematicida ${ }^{10}$.

Tabela 3. Número de juvenis de $2^{o}$ estádio de Meloidogyne incognita e Heterodera glycines (após 48 h +48 h) partindo de um inoculo, contendo 400 nematóides, submetido a diferentes compostos de Mucuna cinerea nas concentrações de 50 e $5 \mu \mathrm{g} \mathrm{mL} \mathrm{m}^{-1}$

Amostras

Mortalidade

\begin{tabular}{|c|c|c|c|c|c|}
\hline & \multirow{2}{*}{$\begin{array}{c}\text { Concentração } \\
\left(\mu \mathrm{gL}^{-1}\right)\end{array}$} & \multicolumn{2}{|c|}{ M. incognita } & \multicolumn{2}{|c|}{ H. glycines } \\
\hline & & $\mathrm{N}^{\circ}$. de Nematóides* & $\%$ & $\mathrm{~N}^{\circ}$. de Nematóides & $\%$ \\
\hline \multirow[t]{2}{*}{ Controle } & 50 & $353,0 \mathrm{a}^{* *}$ & 0,0 & $359,5 \mathrm{a}$ & 0,0 \\
\hline & 5 & $347,0 \mathrm{a}$ & 0,0 & $341,7 \mathrm{a}$ & 0,0 \\
\hline \multirow[t]{2}{*}{1} & 50 & $106,0 \mathrm{e}$ & 70,0 & $325,0 \mathrm{a}$ & 9,5 \\
\hline & 5 & $196,0 \mathrm{c}$ & 43,5 & $335,0 \mathrm{a}$ & 2,0 \\
\hline \multirow[t]{2}{*}{2} & 50 & $152,0 \mathrm{~d}$ & 57,0 & $333,0 \mathrm{a}$ & 7,1 \\
\hline & 5 & $286,0 \mathrm{~b}$ & 17,6 & $322,0 \mathrm{a}$ & 5,8 \\
\hline \multirow[t]{2}{*}{3} & 50 & $247,7 \mathrm{c}$ & 30,0 & $362,7 \mathrm{a}$ & 0,0 \\
\hline & 5 & $290,2 \mathrm{~b}$ & 16,4 & $328,7 \mathrm{a}$ & 3,8 \\
\hline \multirow[t]{2}{*}{4} & 50 & $296,8 \mathrm{~b}$ & 16,2 & $314,7 \mathrm{a}$ & 12,5 \\
\hline & 5 & $321,2 \mathrm{a}$ & 7,5 & $327,2 \mathrm{a}$ & 4,1 \\
\hline \multirow[t]{2}{*}{ CV (\%) } & 50 & - & 14,24 & - & 12,63 \\
\hline & 5 & & 13,37 & & 13,54 \\
\hline
\end{tabular}

* Médias de seis repetições (três contagens por repetição). * As médias seguidas pela mesma letra, para a mesma concentração, são iguais entre si pelo teste de agrupamento de Scott- Knott, a $5 \%$ de probabilidade.

\section{AGRADECIMENTOS}

À Coordenação de Aperfeiçoamento de Pessoal de Nível Superior (CAPES) pela bolsa de estudo de J. C. do Nascimento e ao Conselho Nacional de Desenvolvimento Cientifico e Tecnológico (CNPq) pelas bolsas de pesquisa de A. J. Demuner e L. C. de A. Barbosa e à International Foundation for Science (IFS Grant 2281/1, Suécia) e Fundação de Pesquisa de Minas Gerais (FAPEMIG) pelo apoio financeiro. Ao Dr. O. Howarth (Universidade de Warwick, UK) pela obtenção de alguns dos espectros de RMN. 


\section{REFERÊNCIAS}

1. Buckles, D.; Economic Botany 1995, 49, 13.

2. Tenente, R. C. V.; Lordello, L. G. E.; Dias, J. F. S.; Fitopatologia Brasileira 1984, 9,145 .

3. Siddhuraju, P.; Karuppanan, V.; Janardhanan, K.; J. Agric. Food Chem. 1996, 44, 2636

4. Sharma, R. D.; Pereira, J.; Resck, D. V. S., ed. Em Adubação Verde no Brasil; Fundação Cargill: Campinas, 1984, p. 42-43.

5. Costa, V. S. A.; Pessanha, G. G.; Carvalho, G. M.; Filho, B. R.; Revista Ceres 1995, 42, 584

6. Ishikura, N.; Yoshitama, K.; Phytochemistry 1988, 27, 1555.

7. Fujii, Y.; Shibuya, T.; Yasuda, T.; Japan Agric. Res. Quartely 1992, 25, 238

8. Nogueira, M. A.; Oliveira, J. S.; Ferraz, S.; Phytochemistry 1996, 42, 997.

9. Barbosa, L. C. A.; Barcelos, F. F.; Demuner, A. J.; Santos, M. A.; Nematropica 1999, 29, 81.

10. Barcelos, F. F.; Dissertação de Mestrado, Universidade Federal de Viçosa, Brasil, 1997.

11. Seno, M. S.; Gomes, L.; Cortelazzo, A. L.; Bragantia 1996, 55, 57.

12. Gonzaga, V.; Ferraz, S.; Nematologia Brasileira 1994, 18, 43.
13. Moura, R. M.; Revisão Anual de Patologia de Plantas 1996, 4, 209

14. Weaver, D. B.; Rodriguez-Kábana, R.; Carden, E. L.; J. Nematol. 1993, 25,809 .

15. Paula, V. F.; Barbosa, L. C. A.; Demuner, A. J.; Veloso, D. P.; Howarth, O. W.; Eclética Química 1998, 23, 45.

16. Christie, W. W.; Lipid Analysis, $2^{\text {nd }}$ ed., Pergamon Press: Oxford, 1987.

17. Agrawal, P. K.; Phytochemistry 1982, 31, 3307.

18. The Merck Index, $11^{\mathrm{ed}}, 1991$.

19. McMurry, T. B. H.; Martin, E.; Phytochemistry 1972, 11, 3283.

20. Maiti, B. C.; Thomson, R. H.; Experientia 1976, 32, 1106.

21. Tsai, B. Y.; West, J.; Van Gundy, S. D. Em Phytochemical Pesticides; Kubu, I.; Jacobson, M., eds.; CRC Press: Oxford, 1991, p.1-26.

22. Hasegawa, M.; J. Am. Chem. Soc. 1957, 79, 1738.

23. Hudson, A. T.; Bentley, R.; J. Chem. Soc. Chem. Comm. 1969, 830

24. Markham, K. R.; Mabry, T. J.; Phytochemistry 1968, 7, 791.

25. Letcher, M. R.; Shirley, M. I.; Phytochemistry 1976, 15, 353.

26. Kurosawa, K.; Ollis, W. D.; Sutherland, O. I.; Gottlieb, O. R.; Phytochemistry 1978, 17, 1417.

27. Valle, L. A. C.; Ferraz, S.; Dias, W. P.; Teixeiras, D.; Nematologia Brasileira 1996, 20, 2 . 